\title{
Robotic Antarctic Meteorite Search: Outcomes
}

\author{
Dimitrios S. Apostolopoulos, Liam Pedersen, Benjamin N. Shamah, \\ Kimberly Shillcutt, Michael D. Wagner, William L. Whittaker \\ email: $\{$ da1v, pedersen+, bshamah, kimberly, mwagner, red $\} @$ ri.cmu.edu \\ The Robotics Institute, \\ Carnegie Mellon University \\ Pittsburgh, PA 15213
}

Phone: (412) 268-7224; Fax: (412) 268-1488

\begin{abstract}
:
Automation of the search for and classification of Antarctic meteorites offers a unique case for early demonstration of robotics in a scenario analogous to geological exploratory missions to other planets and to the Earth's extremes. Moreover, the discovery of new meteorite samples is of great value because meteorites are the only significant source of extraterrestrial material available to scientists. In this paper we focus on the primary outcomes and technical lessons learned from the first field demonstration of autonomous search and in situ classification of Antarctic meteorites by a robot. Using a novel autonomous control architecture, specialized science sensing, combined manipulation and visual servoing, and Bayesian classification, the Nomad robot classified five indigenous meteorites during an expedition to the remote site of Elephant Moraine in January 2000. Nomad's expedition proved the rudiments of science autonomy and exemplified the merits of machine learning techniques for autonomous geological classification in real-world settings. On the other hand, the expedition showcased the difficulty in executing reliable robotic deployment of science sensors and a limited performance in the speed and coverage of autonomous search.
\end{abstract}

Keywords: Robotic Meteorite Search, Science Autonomy.

\section{Introduction}

Vast amounts of knowledge and experience are required to autonomously explore a region and identify meteorites from many varieties of terrestrial rocks without real-time input from a field scientist. To accomplish this goal the problem of robotic search for Antarctic meteorites was decomposed into search, detection and classification aspects. These tasks were addressed over a three year period by identifying and testing sensors, developing autonomy and classification software, and demonstrating a rugged mobile robot. The program's first expedition occurred in January of 1998. Component technologies such as a panoramic camera, a ground penetrating radar and instruments for meteorite identification and classification such as a metal detector, magnetometer and reflection spectrometer were individually evaluated in field conditions (Fig. 1). The most important result of the first season was the validation of optical reflection spectroscopy as a valid method of discriminating meteorites from terrestrial rocks [1].

During a second expedition in November 1998, the Nomad robot sought out and classified meteorites in the Patriot Hills region of Antarctica. With the aid of a human assistant to deploy a spectrometer to take readings from

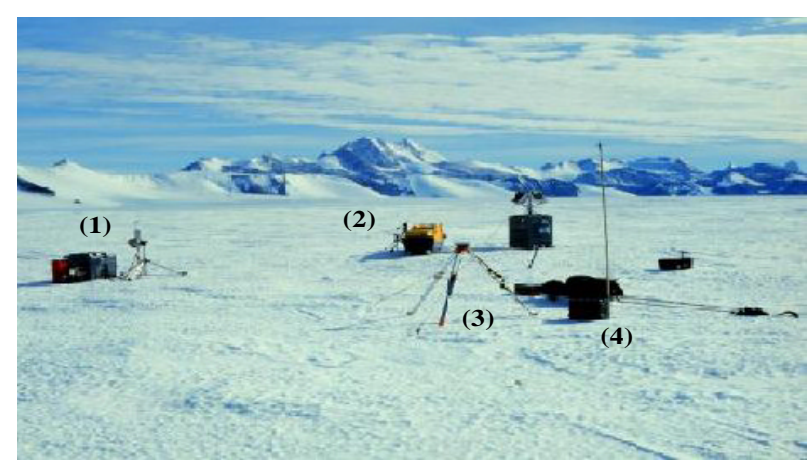

Figure 1: Component technologies evaluated during the first expedition to Antarctica included a panoramic visualization camera (1), a multiband reflection spectrometer (2), GPS (3) and a safeguarding radar sensor (4).

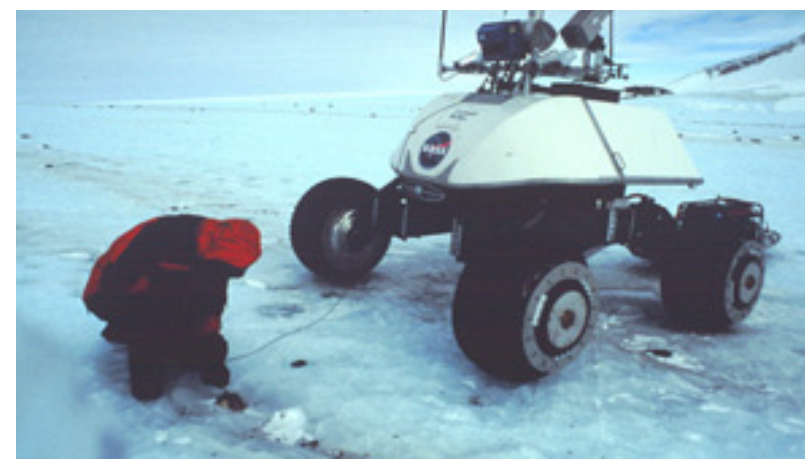

Figure 2: Human assisted rock/meteorite classification during the second Antarctic expedition at Patriot Hills in November 1998. Human deploys the spectrometer, then the robot analyzes the data.

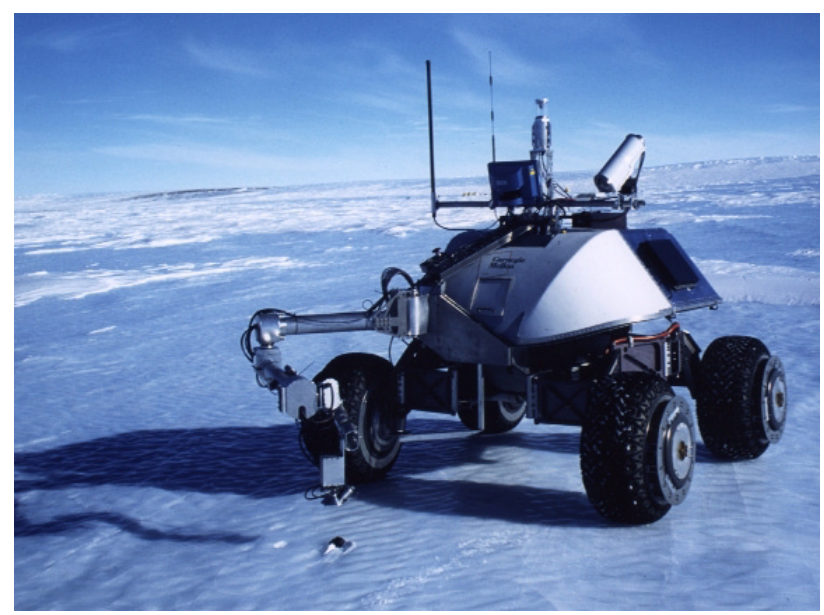

Figure 3: Nomad autonomously investigates a meteorite sample using its manipulator arm at Elephant Moraine, Antarctica, in January 2000. 
rocks, this system classified 50 rocks and 15 planted meteorites (Fig. 2). The robot also autonomously navigated $10 \mathrm{~km}$ of polar terrain using stereo vision, a laser rangefinder and pose sensors [2].

Finally, during a four-week expedition to Elephant Moraine in January of 2000, Nomad accomplished the first robotic meteorite discoveries and performed an unprecedented demonstration of robotic science autonomy (Fig. 3). The main outcome was the classification of five indigenous meteorite samples based on autonomous interpretation of spectral data acquired from indigenous samples. In meeting its primary goal, Nomad explored $2500 \mathrm{~m}^{2}$ of icefields following predetermined search patterns and classified 42 spectra of in-situ rocks. Slow progress due to mechanical and sensing limitations resulted in a small search area over several days of operation. Nomad's rock and meteorite classification capabilities showed marked improvement when retrained on data taken in the field.

This paper first overviews Nomad's mechatronic and cognitive technologies that enabled the autonomous discovery of meteorites. It then details the main outcomes in robotic search and autonomous in situ classification and draws important technical lessons.

\section{Nomad: A Meteorite-Seeking Robot}

Meteorite search and in situ classification was enabled by the Nomad robot, an autonomous planetary-rover prototype with specialized mechatronic and cognitive systems appropriate for this class of polar exploration missions. Nomad's unique combination of in-wheel propulsion, deployable chassis and four-wheel rocker bogie suspension contribute to the robot's high terrainability [3]. Nomad's autonomous navigation system utilizes laser-based range finding and robot pose measurements to detect hazards and safeguard itself [4]. The execution of autonomous science functions is facilitated by a high-resolution camera mounted on the rover's sensor mast, and a manipulator arm which carries an optical spectrometer (Fig. 4).

A 3-DOF SCARA-type (Selective Compliant Articulated Robot for Assembly) manipulator arm is used to deploy and position the primary science sensor, an optical reflection spectrometer [5]. The sensor head located at the tip of the manipulator's wrist accommodates the spectrometer, a camera and two lamps (Fig. 5). The ideal placement of the spectrometer is at 45 degrees to the rock sample surface.

During a search, potential meteorites are identified using a high-resolution 3-chip color CCD camera mounted on a pan / tilt head on Nomad's sensor mast (Fig. 4). Zoom and focus are controlled using custom-designed lens interface hardware. This allows Nomad's autonomy software to capture fully zoomed images of rocks it finds on the ice or snow. During a search the lens is zoomed out to find new rocks. Its maximum field of view is 28 degrees. During
Table 1: Nomad specifications

\begin{tabular}{|ll|}
\hline Physical & \\
\hline Mass: & $725 \mathrm{~kg}$ \\
Power: & $\sim 2000$ watts total continuous \\
Size: & $2.4 \times 2.4 \times 2.4$ m deployed \\
& $0.5 \mathrm{~m} / \mathrm{s}$ maximum \\
Speed: & $0.15 \mathrm{~m} / \mathrm{s}$ nominal \\
\hline Science & $3-\mathrm{CCD}, 640 \times 480,24-$ bit color (on mast) \\
\hline Visual: & CCD 640 x 480 (wrist cam) \\
Spectral: & $300-1100$ nm fiber optic spectrometer \\
Deployment: & 3 DOF manipulator \\
\hline Navigation Sensors & \\
\hline Position estimation: & gyrocompass \\
& GPS \\
& wheel encoders \\
Obstacle avoidance: & single line laser \\
\hline Locomotion & \\
\hline Propulsion: & 4 wheel independent drive \\
Steering: & left / right coordinated \\
\hline Communication & $(1 \mathrm{actuator}$ per side $)$ \\
\hline Data rate: & $1 \mathrm{Mbps}$ \\
Equipment: & $2.4 \mathrm{GHz}$ Wireless Ethernet \\
\hline
\end{tabular}

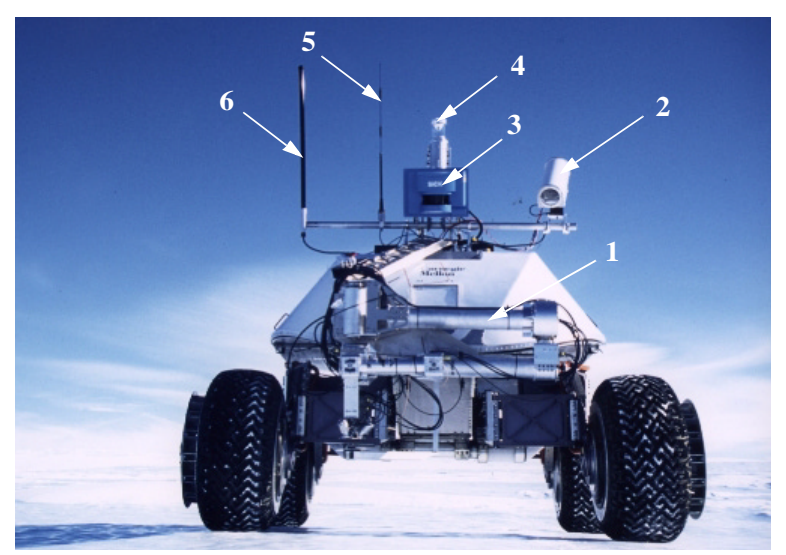

Figure 4: Nomad components: manipulator arm (1), highresolution science camera (2), laser rangefinder (3), panoramic camera (4), radio modem antenna (5) and high-gain wireless ethernet antenna (6).

classification, the lens allows examination of millimeterscale features at a distance of 5 meters.

Nomad deploys its manipulator arm to place a reflection spectrometer within $1 \mathrm{~cm}$ of a sample. Software guides the arm above an estimate of the sample's position. To overcome errors in this estimate, Nomad uses a 92 degree field-of-view camera on the wrist of its arm to perform visual servoing. Once the sample is found in a color image from the arm camera, Nomad can refine its knowledge of the rock's position through a five-step visual servoing process [6]. 


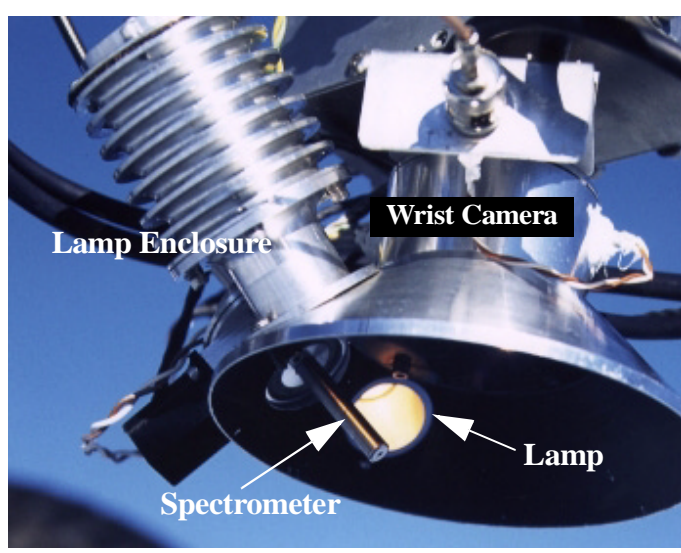

Figure 5: Close-up of Nomad's science sensors mounted on the manipulator's wrist.

Once the manipulator wrist has been servoed to the current sample, a reflection spectrometer obtains spectral data of rock samples. One end of a shielded fiber optic cable is placed close to the rock, while the other end is attached to a reflection spectrometer. Light emitted by lamps on the wrist reflects off the rock and back into a fiber optic cable. Nomad then uses this spectrum to distinguish rocks from meteorites.

\section{Science Autonomy System}

Nomad's Science Autonomy System (SAS) is a control architecture enabling the autonomous discovery and study of rock samples while performing an exhaustive search. The SAS decomposes scientific exploration tasks into directed search, sensor selection and classification tasks. Various modules supervise execution of search patterns, analyze imagery to locate new samples, deploy sensors and classify samples as terrestrial rocks or meteorites

A mission planner module oversees Nomad's autonomous search activities (Fig. 6). An operator issues a command to search an area designated by differential GPS (DGPS) coordinates. In response, the mission planner commands Nomad to navigate search patterns and maintains a list of samples previously located by the target acquisition manager, which segments imagery from the highresolution camera to find new samples. During the search, an obstacle avoidance module maneuvers Nomad away from dangerous terrain [4].

A rock sample is selected for investigation from the mission planner's list using a set of deployment cost parameters such as distance. If a sample is chosen, the mission planner may need to maneuver the robot into the workspace of the appropriate sensor. After maneuvers are complete, sensor calibration, deployment and data acquisition handled by the sensor manager.

Once sensor data have been acquired they are placed in a database for access with the target classifier. This Bayesnetwork classifier computes the posterior probability of the rock under analysis being a meteorite given both

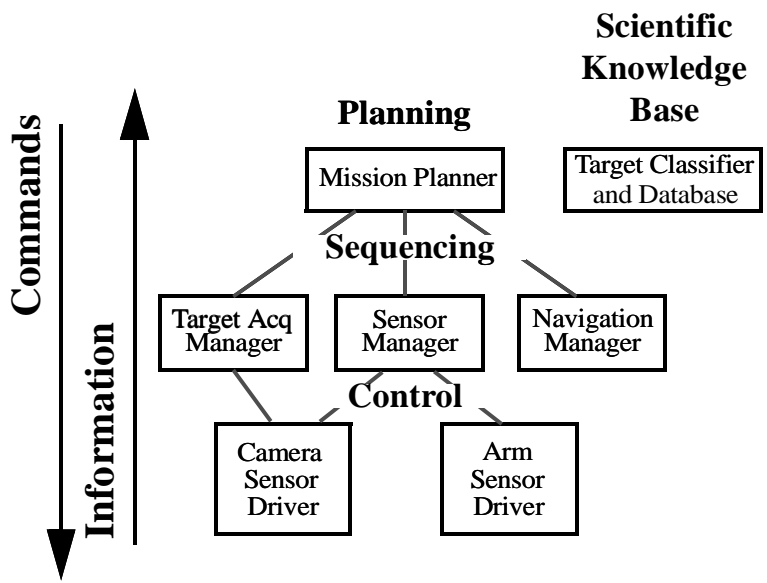

Figure 6: Science Autonomy System as a modified threetiered architecture.

imagery and spectral data [7]. Image features used include color and size. A fixed set of spectral features is matched against Gaussian templates; a positive normalized coefficient indicates a reflection peak, while negative values indicates a trough. The classifier differentiates between general types of rocks, such as sedimentary, metamorphic, igneous, extraterrestrial or "other," (generally meaning ice or snow) as well as between specific mineral types such as limestone, granite or stony iron meteorite [1].

Should the target be given a high probability of being a meteorite, Nomad waits for human validation. If there is another target within the manipulator's workspace, the robot stays in its location and repeats the sensor deployment and classification cycle. Otherwise, the mission planner maneuvers Nomad to the location of the next target in the list or resumes its search pattern to seek new targets. The autonomous search is complete when Nomad covers the designated area and classifies all identified targets.

For a detailed discussion of Nomad's Science Autonomy System please refer to the companion paper [8].

\section{Robotic Meteorite Search Demonstration}

Nomad's robotic technologies were validated during a field expedition to the remote site of Elephant Moraine in January 2000.

Elephant Moraine, located at $76 \operatorname{deg} 16^{\prime} \mathrm{S}, 157 \mathrm{deg} 12^{\prime} \mathrm{E}$ is a barren region of Antarctica that is known to contain numerous meteorites. The site is located approximately 160 miles northwest of McMurdo Station, the logistical center of Antarctica. The field team landed in McMurdo in late December, 1999. Supplies for the field expedition to Elephant Moraine were collected and staged by support personnel. The McMurdo area also provided an environment in which to test Nomad's capabilities [6]. Functions never previously tested in Antarctica were validated, such as target acquisition and visual servoing of the manipulator end-effector to a rock target. Nomad and 
the field team consisting of six robotics researchers and one meteorite expert were airlifted to Elephant Moraine on January 7 and January 10, respectively.

\section{Outcomes and Lessons}

During the ten demonstrations performed at Elephant Moraine, a total area of approximately $2500 \mathrm{~m}^{2}$ was searched in 16 hours spread over 10 days. Three meteorites were found and correctly classified during autonomous searches, out of a total of 42 targets examined. Figure 7 and Table 2 summarize Nomad's activity during these demonstrations. An additional two meteorites were correctly classified during tests performed without patterned searches (Fig. 8).

\subsection{Science Sensor Deployment}

Nomad's manipulator was deployed successfully on 42 out of 63 attempts not manually aborted (72\% success rate). Unfortunately the touch probe mounted on the wrist, which was designed to halt the wrist when it reached the proper height above a sample, failed in the cold environment (all other mechanisms functioned reliably even in extremely cold conditions). Teleoperation from the base camp was required to safely guide the wrist down for the final $10 \mathrm{~cm}$ to the sample and was performed by viewing wrist camera images from the base camp and verifying downward movement steps with an observer in the field. Not considering this problem, 13 of the successful attempts required some other type of human intervention. Failures generally involved the spectrometer being grossly misaligned with the rock, causing the system to acquire meaningless spectra [6].

During meteorite searches, the SAS had no knowledge of sensor data quality. Often the spectrometer was not properly positioned over rock samples, either missing them completely or approaching them at a bad angle to the rock's surface. There was no external measure of confidence in the data; although the arm visual servoing algorithm was effective there was no reliable method of sensing the safe and ideal final height of the sensor head. In theory, the Bayes-based target classifier has the capability to recognize outliers, but cannot discriminate between those due to unusual rocks and those due to bad readings.

An automated system to verify correct sensor placement is essential for both proper classification and to inform the mission planner that more data is needed. It would provide a measure of confidence that the data being classified were valid. The sensor head must therefore have some form of closed-loop control routine that receives feedback on the position of the spectrometer relative to a rock sample. This represents a difficult problem because of space constraints on the sensor head, the variable geometry of rocks and the sensitivity of the spectrometer.

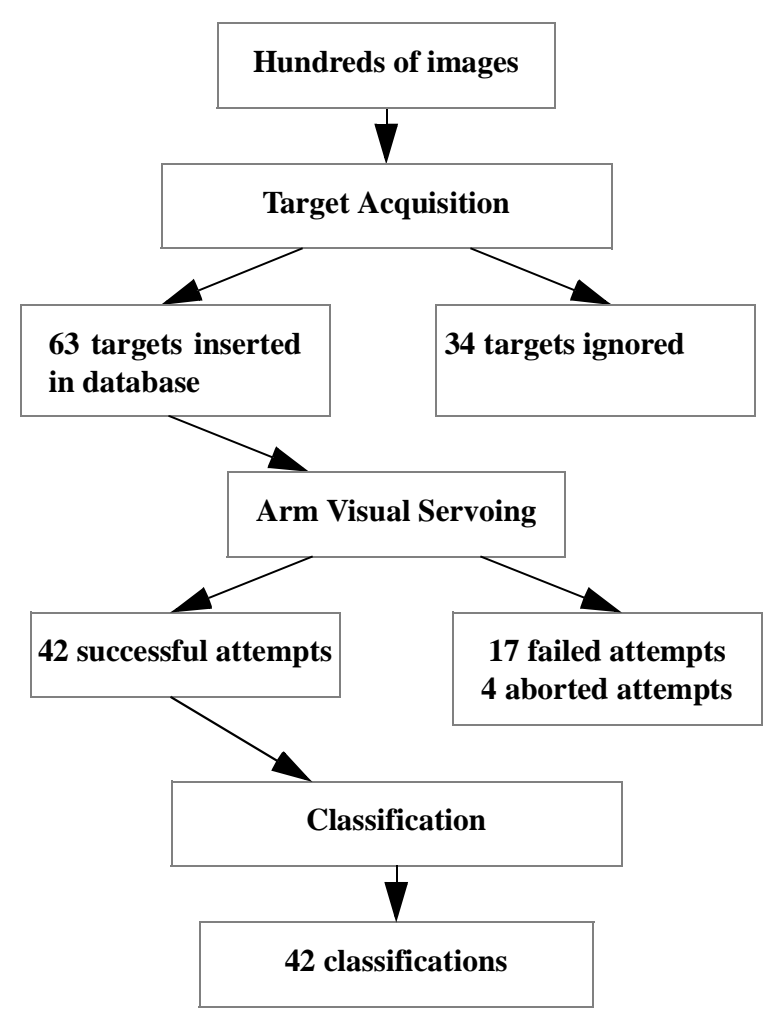

Figure 7: Target acquisition and classification results.

\begin{tabular}{|l|l|}
\hline \multicolumn{1}{|c|}{ Item } & \multicolumn{1}{c|}{ Results } \\
\hline \hline \multirow{3}{*}{ Patterned Search } & $\begin{array}{l}2500 \mathrm{~m}^{2} \text { searched in 16 hours } \\
37 \% \text { of time spent searching } \\
47 \% \text { of time spent deploying sensors } \\
6 \% \text { of time spent maneuvering robot to target }\end{array}$ \\
\hline & $\begin{array}{l}5 \text { meteorites correctly classified } \\
22 \text { rocks correctly classified } \\
17 \text { misclassifications } \\
42 \text { total spectra classified }\end{array}$ \\
\hline Slassification & $\begin{array}{l}\text { Two 10 W panels recorded solar irradiation } \\
\text { continuously }\end{array}$ \\
\hline Weather Data & $\begin{array}{l}\text { Temperature and wind speed recorded contin- } \\
\text { uously }\end{array}$ \\
\hline Panoramic Cam & 42 images taken \\
\hline $\begin{array}{l}\text { Public Participa- } \\
\text { tion }\end{array}$ & $\begin{array}{l}\text { Daily download of 1-2 MB recorded data to } \\
\text { publicly accessible web site }\end{array}$ \\
\hline
\end{tabular}

Table 2: Nomad's performance at Elephant Moraine.

\subsection{Autonomous Rock/Meteorite Classification}

A problem with the camera zoom control prevented the acquisition of close up color images of samples, so classification had to be done with spectra only. Overall performance is indicated by the rate of classification (ROC) performance curves in Figure 9: These were generated from a test set of rocks and meteorites in the moraine, some of which were analyzed multiple times. Curve (i) is plotted using the classification output of the $a$ priori classifier built from laboratory data. There is a notable improvement in curve (ii), the ROC curve for the 
same classifier further trained with field data from 8 rocks and 2 meteorites not in the test set.
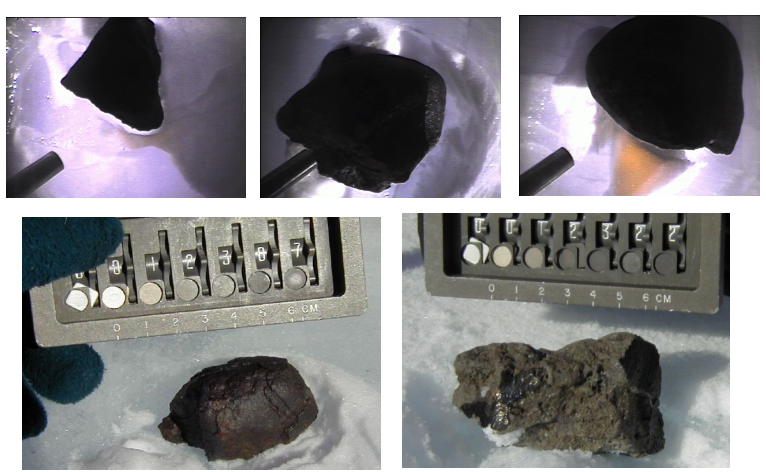

Figure 8: Nomad's meteorite finds: The top row shows three ordinary meteorite types found and classified on January $22 / 25 / 26$, respectively. The images were acquired by the wrist camera during placement of the spectrometer probe (visible in all three images). The bottom row shows the fourth ordinary meteorite found on January 27 and a special type found on January 29. Nomad classified the fifth rock as "interesting". Nomad's classifier had been trained on this rare type only once before using a sample provided by the Smithsonian Institution.

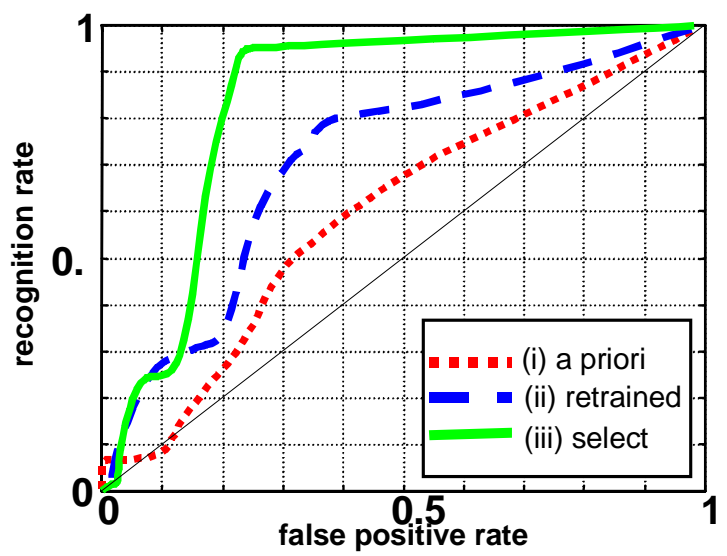

Figure 9: Rate of classification curves based on data acquired by Nomad in Antarctica.

Even with retraining, classification is systematically bad for hydro-thermally altered dolerites and basalts, a particular class of rocks that were found in Elephant Moraine. These rocks are stained red with iron oxide (rust) whose spectrum has a prominent peak at $900 \mathrm{~nm}$, precisely where many meteorite spectra also have a peak. This is not surprising, given that most meteorites contain metallic iron, and therefore can have surface rust. Unfortunately, these rocks were absent from the initial training set and not initially expected in this area. Performance is much better if these rocks are removed from the test set (Fig. 9, curve (iii)) and the retrained classifier is used.

The classification results make one lesson clear: there is no substitute for training sets from the field. While this amount of prior knowledge is not possible in entirely new environments, classification improves with additional learning in the field. A period of in situ training with human expert supervision could prove quite worthwhile for extended autonomous exploration.

\subsection{Autonomous Patterned Search}

A ground plot of all the locations Nomad visited during the expedition is shown in Figure 10. Nomad traveled from the base camp to the southern moraine location on January 19, and then to meteorite site 1 on January 20. Nomad returned to base on January 23, and then went on to meteorite site 2 on January 24. Nomad drove to the northern moraine location, via the base camp, on January 26-27, and returned to base on January 30.
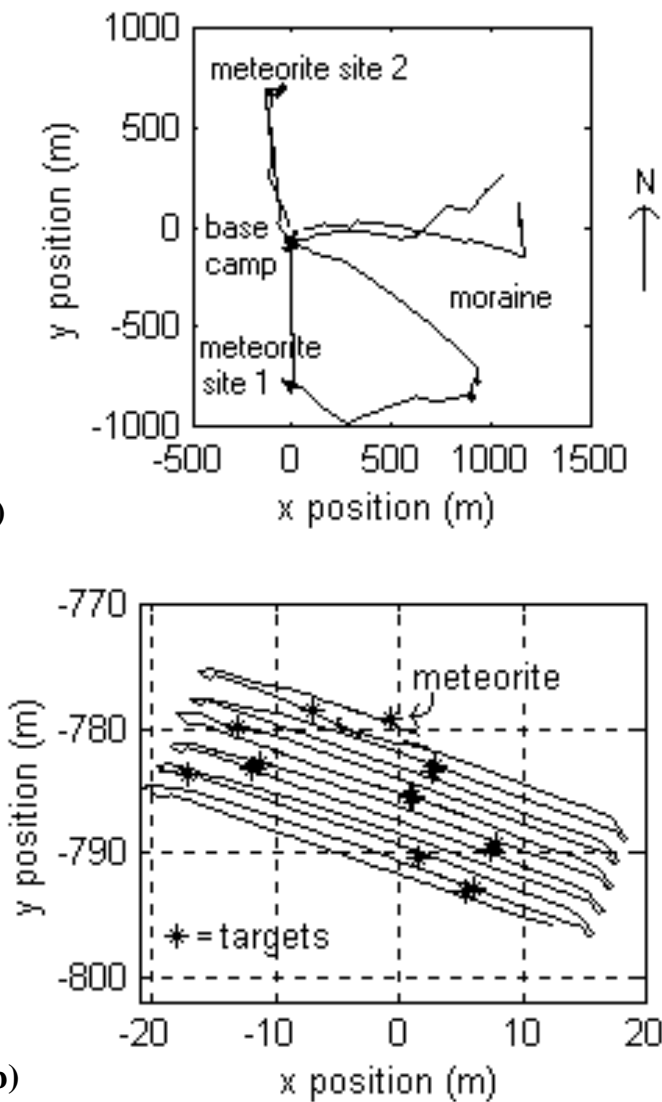

Figure 10: DGPS plots of Nomad's: (a) overall expedition search paths, (b) patterned search leading to the discovery of the first meteorite.

A close-up view of the first meteorite search demonstration area is shown in Figure 10 (b), where three searches took place from January 20-22. In the third search, 15 rock targets were investigated, one of which was successfully identified as a meteorite.

Nomad's search efficiency was limited by two major factors. First, the operating speed was slow. Even if the robot never paused to analyze a rock it could only travel $0.5 \mathrm{~km}$ in an hour. The manipulator arm also moves slowly, both because of a low velocity and because only one joint was actuated at a time. The mechanical speed limit of this system has clearly not been explored. 
The second major factor limiting Nomad's search efficiency was target acquisition. The high-resolution camera's maximum field of view was only 28 degrees, and the camera was pointed at the ground approximately $2.5 \mathrm{~m}$ in front of the robot. This results in a maximum search area of around $800 \mathrm{~m}^{2} / \mathrm{hr}$ if the robot never pauses to deploy its arm. Search productivity could be improved by using target acquisition sensors with a wider field of view. The ability to search wider rows not only allows more targets to be viewed but also decreases the total distance traveled by the robot.

Finally, the current system for spectrometer deployment is a time intensive operation. By improving the target acquisition phase to discriminate rocks that have a higher probability of being a meteorite much less time would be spent deploying the spectrometer. For instance, in areas near moraines, the robot spent approximately $50 \%$ of its time deploying its manipulator to take spectra of a large number of rocks. Some of these rocks were clearly not meteorites due to their size and color. Unfortunately, the bright sunlight reflecting off the ice closed the lens' autoiris giving most rocks a black appearance. The classifier uses black coloring as an indication of a meteorite, so this problem caused rocks to be incorrectly classified as meteorites. Were it not for this problem, many rocks should have been quickly judged to be terrestrial in origin.

\section{Conclusions}

Through the discovery and in situ classification of Antarctic meteorites Nomad demonstrated rudimentary robotic capabilities for autonomous scientific exploration. The implementation of the SAS control architecture that prioritizes science mission goals to guide search and in situ operations was critical to Nomad's success. Complete coverage through patterned search proved to be an effective model for meteorite search but requires a priori identification of promising areas to search. Slow progress due to mechanical and operational limitations resulted in a small search area over several days of operation. Another reason for the slow pace was the fact that Nomad classified every rock on its path using both vision to identify the rock target and spectroscopy for classification. Selective utilization of sensors based on utility and ease of deployment should have a profound improvement on the speed of autonomous search.

Bayes network-based classification has proved to be a viable method for autonomous meteorite classification. However, a method for autonomous data quality assessment is necessary to ensure the trustworthiness of information given to the classifier. Moreover, an autonomous science data collection system must handle sensor recalibration and mitigate the effects of the environment on the quality of the readings.

Future scientific missions could clearly benefit from Nomad's technologies. Robust, high-speed, autonomous robots imbued with science autonomy and sensors like
Nomad's could perform geological classification missions in remote polar regions and other planetary analogous environments. The envisioned robotic autonomy could enable pioneering discovery missions such as search for life at the Earth's extremes and beyond.

\section{Acknowledgments}

This program has been supported by the National Aeronautics and Space Administration (NASA) under grants NAG5-7707 and NAG9-1090. The authors would like to the thank Melvin Montemerlo, David Lavery and Chris Culbert of NASA for their long-standing support of this work. We would also like to thank the National Science Foundation (NSF) and Antarctic Search for Meteorites Project (ANSMET) for providing scientific guidance, field expertise and excellent logistical support. We are especially appreciative of the contributions of Scott Borg, Ralph Harvey, John Schutt and William Cassidy.

This work would not have been possible without the dedicated efforts of the Nomad Meteorobot team. Thank you all for a wonderful endeavor.

\section{References}

[1] Pedersen, L., Apostolopoulos, D., Whittaker, W. L., Benedix, G., and Roush, T., "Sensing and Data Classification for Robotic Meteorite Search", Proceedings of the 1998 SPIE International Conference on Mobile Robots and Intelligent Transportation Systems, pp.157 - 167, Boston, MA, November 1998.

[2] Apostolopoulos, D., Wagner, M., and Whittaker, W. L., "Technology and Field Demonstration Results in the Robotic Search for Antarctic Meteorites", Proceedings of the International Conference on Field and Service Robotics, pp.185 190, Pittsburgh, PA, August 29-31, 1999.

[3] Shamah, B., Apostolopoulos, D., Rollins, E., and Whittaker, W. L., "Field Validation of Nomad's Robotic Locomotion", Proceedings of the 1998 SPIE International Conference on Mobile Robots and Intelligent Transportation Systems, pp. 214 222, Boston, MA, November 1998.

[4] Moorehead, S., Simmons, R., Apostolopoulos, D., and Whittaker, W., L., "Autonomous Navigation Field Results of a Planetary Analog Robot in Antarctica", Proceedings of the 1999 International Symposium on Artificial Intelligence, Robotics and Automation in Space, pp. 237 - 242, Noordwijk, Holland, June 1999.

[5] Spong. M., and Vidyasagar, M., Robot Dynamics and Control, New York, NY, John Wiley, 1989.

[6] Wagner, M., "Experimenter's Notebook: Robotic Search for Antarctic Meteorites 2000 Expedition", Technical Report CMURI-TR-00-13, Robotics Institute, Carnegie Mellon University, June, 2000.

[7] Cheeseman, P., Kelly, J., Self, M., Stutz, J., Taylor, W., and Freeman, D., "AutoClass: A Bayesian Classification System", Proceedings of the Fifth International Conference on Machine Learning, pp. 54-64, Ann Arbor, MI, 1988.

[8] Wagner, M., Apostolopoulos, D., Shillcutt, K., Shamah, B., Simmons, R., and Whittaker, W. L., "The Science Autonomy System of the Nomad Robot", in International Conference for Robotics and Automation, Seoul, South Korea, May 2000. 